\title{
Editorial
}

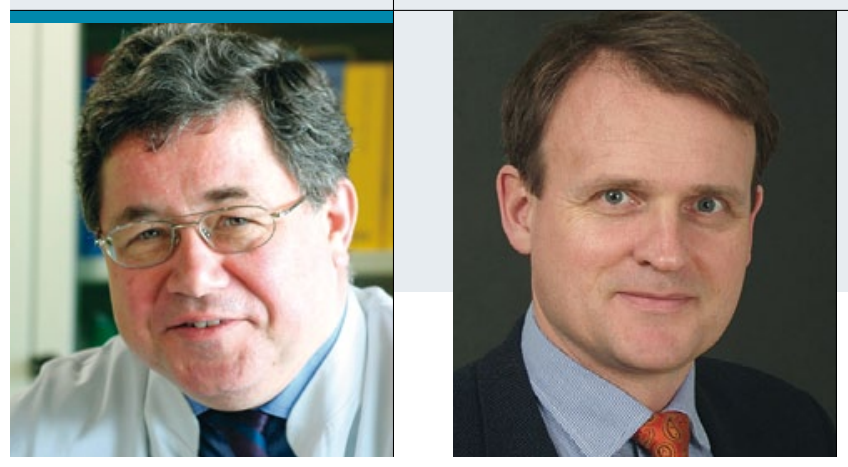

"Gerade in der Berufsdermatologie

spielt der Epikutantest in der Diagnostik

eine zentrale Rolle."

Prof. Dr. Hans F. Merk, Hautklinik der Medizinischen

Fakultät, Universitätsklinikum der RWTH Aachen

Prof. Dr. Thilo Jakob, Allergieabteilung und Forschergruppe

Allergologie, Universitäts-Hautklinik Freiburg

\section{Gefahr gebannt, Epikutantest gerettet!}

$\mathrm{P}$

ositives ist zu vermelden: Noch vor wenigen Monaten wurde in diesem Heft über bedenkliche Erschwernisse bei der Fortentwicklung des Epikutantests und die epidemiologische Erfassung von Kontaktallergenen - wie sie in Deutschland vor allem durch das Information Network of Departments of Dermatology (IVDK) in Göttingen erfolgen - berichtet. In der Überschrift wurde die Gefahr: „Der Allergie-Diagnostik mit dem Epikutantest droht das Aus" herausgestellt [Schnunch A et al. Allergo J 2012; 21: 68-9]. Diese Situation erschien insbesondere auch vor dem Hintergrund fehlender Tierexperimente bei der Entwicklung neuer Kosmetika ab 2013 besonders bedenklich. Der Grund dafür war eine Novellierung der „Arzneimittel- und Wirkstoffherstellungsverordnung“, die in Verbindung mit $\$ 4$ (5) des Arzneimittelgesetzes (AMG) Kontaktallergene als Diagnostika bzw. Arzneimittel definiert und damit Studien und Neuentwicklungen mit ähnlich hohem Aufwand wie bei Medikamenten durchgeführet werden müssten. Zudem müssten die Substanzen Reinheitsgrade aufweisen, die gerade zum Allergennachweis in nicht wenigen Fällen abträglich sind, da Kontaktallergene auch durch Sensibilisierung gegen Verunreinigungen in entsprechenden Berufsstoffen etc. bestehen können.

Dank vielfältiger Aktivitäten auf nationaler und europäischer Ebene vor allem der Kollegen Prof. Dr. Sven Malte John, Osnabrück, und Prof. Dr. Axel Schnuch, Göttingen, zusammen mit den betroffenen Fachgesellschaften wurde kürzlich zu einem Gespräch durch das Paul-Ehrlich-Institut eingeladen, an dem alle interessierten Behörden und Verbände beteiligt wurden. Im Ergebnis führte dieses Gespräch zu einer Lösung der Problematik: Epidemiologische Erhebungen können - nach Zustimmung der Ethikkommissionen - nun weiterhin mit angemessenem Aufwand durchgeführt werden, da epidemiologische Studien per se nicht dem AMG unterliegen. Auch
Studien mit neuen möglichen Kontaktallergenen können in einem Rahmen durchgeführt werden, der im Verhältnis zum Produkt steht. Dieses Ergebnis ist im Besonderen auch für die Berufsdermatologie von großer Bedeutung. Gerade hier spielt der Epikutantest in der Diagnostik eine zentrale Rolle und muss insbesondere bei diversen Fragestellungen mit unbekannten bzw. wenig definierten Substanzen durchgeführt werden. Zudem besitzt in der Berufsdermatologie auch die epidemiologische Auswertung besondere Bedeutung in der Prävention.

Dem Thema Beruf und Allergie sind zwei Beiträge in dieser Ausgabe gewidmet: Es ist das besondere Verdienst von Kollege Dr. Heinrich Dickel, in den vergangenen Jahren die Bedeutung des Epikutantests bei der Modifikation mittels Abrissverfahren und damit bei der Reduktion der Hautbarriere sowohl auf klinischer wie auch auf wissenschaftlicher Ebene ausgearbeitet zu haben. Dickel stellt in seiner Kasuistik praktische Beispiele für die Bedeutung des Epikutantests in der Berufsdermatologie vor (S. 348). Besondere Aspekte der berufsbedingten Allergien der Atemwege werden im Rahmen des CME-Beitrags vorgestellt (S. 354).

Schließlich führen Prof. Dr. Claudia Traidl-Hoffmann und Dr. Stefanie Gilles Sie ab Seite 344 in unsere neue Serie „Das kleine 1 x 1 der Immunologie“ ein, die ab sofort im jeder Ausgabe von Allergo Journal erscheinen wird. Es folgt direkt danach der erste Teil der Serie, der sich mit der physiologischen und pathophysiologischen Bedeutung dendritischer Zellen beschäftigt (S. 346).

Wir wünschen Ihnen viel Spaß beim Lesen!

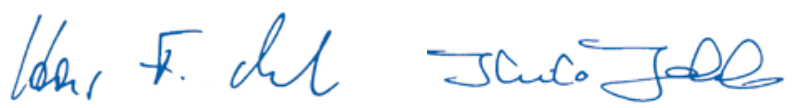

